\title{
A SYNTHETIC THEORY of MOTION for TITAN-HYPERION
}

\author{
L. DURIEZ
}

Laboratoire d'Astronomie de l'Université des Sciences et Techniques de Lille-Flandres-Artois, 1 Impasse de l'Observatoire, F 59000 Lille, France

\begin{abstract}
We present an iterative method allowing to synthetize a semi-numerical solution for the equations of motion of the resonant Saturn's satellites Titan-Hyperion (limited now to the planar problem). The current theory of Hyperion by Taylor, Sinclair \& Message (1987) gives the greatest terms of the long-period part of the solution (depending on two angles: the libration angle $\tau$, and the angular distance of the pericenters $\zeta$ ). Using it as a first approximation, this solution is substituted numerically in the exact Lagrange equations of motion for Titan and Hyperion, computed for many values of the three angles : $\tau, \zeta$ and $\phi$ (the mean synodic longitude). Then, a multivariable Fourier transform allows to reconstruct the equations in these three angles, that is in same form as the initial one with, in addition, the short-period terms. Then, a solution may be obtained and used as a better approximation in an iterative process. Besides a complete determination of the short-period perturbations of Hyperion obtained here completely for the first time, some long-period perturbations of Titan by Hyperion are also found which would be non negligible at the $10 \mathrm{~km}$ level.
\end{abstract}

Key words: Titan-Hyperion - semi-numerical theory - resonance

\section{Introduction}

The orbital motion of Hyperion around Saturn is strongly disturbed by the resonance 3:4 with Titan, characterized by : $3 N^{\prime}-4 N+\langle\dot{\varpi}\rangle=0^{1}$. Then, observation shows that the critical angle : $\theta=3 \lambda^{\prime}-4 \lambda+\varpi=3\left(\lambda^{\prime}-\lambda\right)-M$ librates around $180^{\circ}$ with a large amplitude : $\theta=180^{\circ}-36^{\circ} .5 \sin \tau-13^{\circ} .5 \sin \zeta+\cdots$, where $\tau$ and $\zeta$ are two long-period arguments whose periods are about $640 \mathrm{~d}$ and $6850 \mathrm{~d}$ respectively. $\tau$ is the argument of the libration, while $\zeta$ represents a linear function of time such that $\dot{\zeta}=\left\langle\dot{\varpi}-\dot{\varpi}^{\prime}\right\rangle$. When restricted to the plane problem, the representation of the motion depend on these two arguments and on the synodic angle $\phi$, also linear function of time defined by $\dot{\phi}=N^{\prime}-N$; the period of $\phi$ is about $63 \mathrm{~d}$. This last argument may be considered as a short-period one.

The main previous works about the Hyperion's motion are :

- Woltjer (1928) who constructed numerically a first order semi-numerical representation of the orbital elements including long-period terms only; Woltjer computes numerically the influence of the short-period terms directly on positions for the date of each available observation.

- Taylor et al.(1987) who made a new fit of the Woltjer's theory to observations and then obtained the short-period perturbations on orbital elements, from an analysis of the residuals obtained between the fitted long-period theory and a complete numerical integration; however, their discretisation allow them to find the only short-period terms whose period is longer than 30 days.

- Message (1989) who proposed a second order long-period analytical theory in which the short-period terms which are necessary to know at the first order to

1 Along this text, $a, e, N, \lambda$, $\varpi$ and $M$ stand for semi-major axis, eccentricity, mean mean motion, mean longitude, longitude of pericentre and mean anomaly respectively. The primed quantities concern Titan. $\langle x\rangle$ stands for the mean value of $x$ over time. 
compute the second order, are not explicited : their influence is only computed by numerical averaging.

- Vienne \& Duriez (1991) who used a technique of synthetic expansions to compute directly all the short-period perturbations of the orbital elements of Hyperion at the first order in the Titan's mass. It appears there that many terms with period shorter than 30 days have not to be neglected.

The present work is an extension of that presented in Vienne \& Duriez (1991) : The process described there is now iterated, allowing to obtain synthetic expansions of both long-period and short-period terms up to a given precision. Assuming that Hyperion has a mass equal to $310^{-8}$ that of Saturn ${ }^{2}$, we have also computed at the same time the perturbations of Titan by Hyperion.

\section{Synthetic expansions}

The details of the method used to produce synthetic expansions of the Lagrange equations is given in (Vienne \& Duriez, 1991, Sect. 4). Say only here that the plane motion of the pair Titan-Hyperion may be represented by the following series depending on $\theta_{i j k}=i \phi+j \tau+k \zeta$ :

$$
\begin{aligned}
& \lambda=\lambda_{0}+N t+\delta \lambda \quad \text { with } \quad \delta \lambda=\sum_{i, j, k} \lambda_{i j k} \sin \theta_{i j k} \\
& \varpi=\varpi_{0}+\langle\dot{\varpi}\rangle t+\delta \varpi \quad \delta \varpi=\sum_{i, j, k} \varpi_{i j k} \sin \theta_{i j k} \\
& e=e_{0}+\delta e \\
& a=a_{0}+\delta a
\end{aligned}
$$

At the begining, only the main terms of each series are known from current theories (for example, Taylor et al., 1987) and have long periods. We give here some of them : $\lambda_{010}=9^{\circ} .13, \varpi_{001}=-13^{\circ} .47, e_{0}=0.1046, e_{001}=0.0242, a_{0}=1.4810^{6} \mathrm{~km}$, $a_{010}=0.0035 a_{0}$. Then, the other terms are synthesized from them, like the shortperiod terms presented in Vienne \& Duriez (1991). In this aim, assuming analogous expressions for Titan, we define the angles $u_{1}, u_{2}$ and $u_{3}$ :

$$
\begin{aligned}
& u_{1}=\lambda^{\prime}-\lambda=\phi+\delta \lambda^{\prime}-\delta \lambda \\
& u_{2}=\theta=180^{\circ}+3 \delta \lambda^{\prime}-4 \delta \lambda+\delta \varpi \\
& u_{3}=\varpi-\varpi^{\prime}=\zeta+\delta \varpi-\delta \varpi^{\prime}
\end{aligned}
$$

from which we may obtain $\lambda, \lambda^{\prime}$ and $\varpi$ as functions of $\phi, \tau$ and $\zeta$ :

$$
\begin{aligned}
& \lambda=\varpi^{\prime}+3 u_{1}-u_{2}-u_{3} \\
& \lambda^{\prime}=\varpi^{\prime}+4 u_{1}-u_{2}-u_{3} \\
& \varpi=\varpi^{\prime}-u_{3}
\end{aligned}
$$

$\varpi^{\prime}$ may remain arbitrary; in the following, we denote by $x$ any of the elements $\left\{a, e, a^{\prime}, e^{\prime}\right\}$ and by $y$ any of $\left\{\lambda, \varpi, \lambda^{\prime}, \varpi^{\prime}\right\}$. Therefore, $x$ and $y$ are expressions in the form :

$$
x=x_{0}+\sum_{i j k} x_{i j k} \cos \theta_{i j k} \quad y=y_{0}+\dot{y}_{0} t+\sum_{i j k} y_{i j k} \sin \theta_{i j k}
$$

2 The Hyperion's mass has been estimated from its dimensions (ellipsoid $205 \times 130 \times 110 \mathrm{~km}$ ), and with a density equal to $1.4 \mathrm{~g} \cdot \mathrm{cm}^{-3}$ (average value of the densities of other small satellites). 
Hence, from expressions (1), (2) and (3), we can compute $x$ and $y$ for any value of $\phi, \tau$ and $\zeta$, as well as the right-hand members of the Lagrange equations for Titan and Hyperion by using the formula given by Chapront et al.(1975). $p_{\phi}, p_{\tau}$ and $p_{\zeta}$ being given integer, we compute these right-hand members at the 'harmonic abscissae' : $\left\{\phi_{i}=2 i \pi / p_{\phi}\right\}_{i=0 \cdots p_{\phi}},\left\{\tau_{i}=2 i \pi / p_{\tau}\right\}_{i=0 \cdots p_{\tau}},\left\{\zeta_{i}=2 i \pi / p_{\zeta}\right\}_{i=0 \cdots p_{\zeta}}$. Hence, a 3-dimensional FFT routine allows to synthesize the Lagrange equations as semi-numerical expansions in the three angles $\phi, \tau$ and $\zeta$, that is in the form :

$$
\left(\frac{d x}{d t}\right)_{s y n t h}=-\sum_{i j k} X_{i j k} \sin \theta_{i j k} \quad\left(\frac{d y}{d t}\right)_{s y n t h}=\sum_{i j k} Y_{i j k} \cos \theta_{i j k}
$$

\section{Iterative process}

By derivation of expressions (4), we have also :

$$
\left(\frac{d x}{d t}\right)_{d e r i v}=-\sum_{i j k} x_{i j k} \dot{\theta}_{i j k} \sin \theta_{i j k} \quad\left(\frac{d y}{d t}\right)_{d e r i v}=\sum_{i j k} y_{i j k} \dot{\theta}_{i j k} \cos \theta_{i j k}
$$

If $x$ and $y$ are solutions of the Lagrange equations, the expressions (5) must match those (6). In fact, at the begining, it is not true; the frequencies $N, N^{\prime},\langle\dot{\varpi}\rangle$ and $\left\langle\dot{\varpi}^{\prime}\right\rangle$ being supposed known and fixed to the values given by current theories, we have to adjust the other parameters of the problem, which are some of the $x_{i j k}$ and $y_{i j k}$, mainly : $a_{0}, a_{0}^{\prime}, e_{0}, e_{0}^{\prime}$ and $\lambda_{010}$ (amplitude of the libration in $\lambda$ ). Denoting these parameters by $c_{l}$ and varying numerically each one, we can compute the value of each partial derivative of $X_{i j k}$ and $Y_{i j k}$ with respect to $c_{l}$, to obtain, instead of (5) :

$$
\begin{aligned}
& \left(\frac{d x}{d t}\right)_{s y n t h}=-\sum_{i j k}\left(X_{i j k}+\sum_{l} \frac{\partial X_{i j k}}{\partial c_{l}} \Delta c_{l}\right) \sin \theta_{i j k} \\
& \left(\frac{d y}{d t}\right)_{s y n t h}=\sum_{i j k}\left(Y_{i j k}+\sum_{l} \frac{\partial Y_{i j k}}{\partial c_{l}} \Delta c_{l}\right) \cos \theta_{i j k}
\end{aligned}
$$

Hence, we find a new solution $x^{\prime}$ and $y^{\prime}$ by identifying :

$$
x_{i j k}^{\prime}=\left(X_{i j k}+\sum_{l} \frac{\partial X_{i j k}}{\partial c_{l}} \delta c_{l}\right) / \dot{\theta}_{i j k} \quad y_{i j k}^{\prime}=\left(Y_{i j k}+\sum_{l} \frac{\partial Y_{i j k}}{\partial c_{l}} \delta c_{l}\right) / \dot{\theta}_{i j k}
$$

where the $\delta c_{l}$ are the least-square solution of the system :

$$
\left(x_{i j k}+\delta x_{i j k}\right) \dot{\theta}_{i j k}=X_{i j k}+\sum_{l} \frac{\partial X_{i j k}}{\partial c_{l}} \delta c_{l} ; \quad\left(y_{i j k}+\delta y_{i j k}\right) \dot{\theta}_{i j k}=Y_{i j k}+\sum_{l} \frac{\partial Y_{i j k}}{\partial c_{l}} \delta c_{l}
$$

In this system, when $x_{i j k}$ or $y_{i j k}$ correspond to some $c_{l}$, the $\delta x_{i j k}$ or $\delta y_{i j k}$ is identified to $\delta c_{l}$, and else it is zero. This resolution depends also on the constraints : $\langle d \lambda / d t\rangle=N,\left\langle d \lambda^{\prime} / d t\right\rangle=N^{\prime},\left\langle d\left(3 \lambda^{\prime}-4 \lambda+\varpi\right) / d t\right\rangle=0$, and accounts on the secular perturbations of Titan and Hyperion by all other satellites and by the Saturn's oblateness.

Hence, the new solutions $x^{\prime}$ and $y^{\prime}$ may be used instead of the previous $x$ and $y$ to iterate the process up to stabilization. 

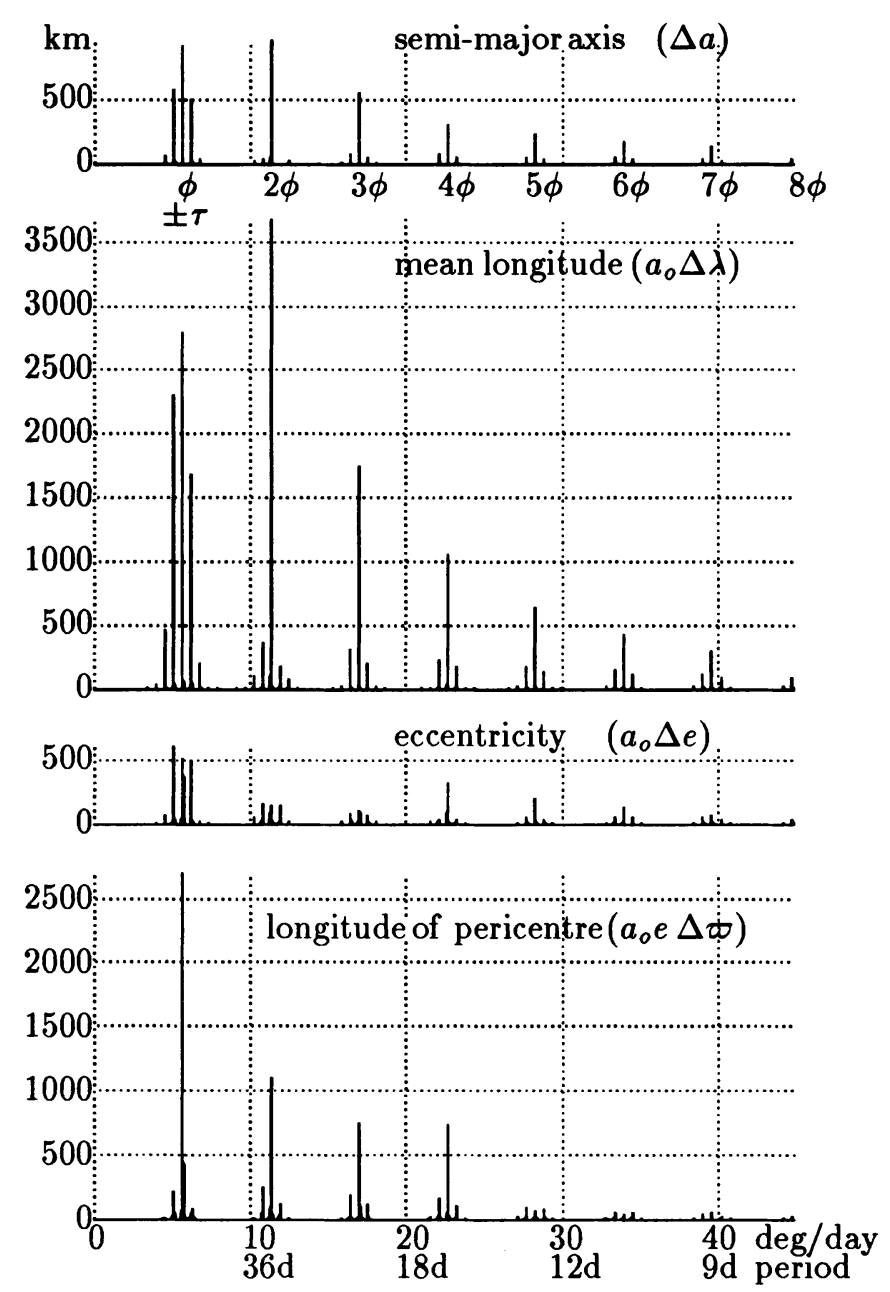

Fig. 1. Short-period perturbations of Hyperion; the smallest lines represent about $15 \mathrm{~km}$ and remind that $700 \mathrm{~km}$ correspond to 0.1 arcsec as seen from Earth at oppositions.

\section{Application}

In practice, we have used $p_{\phi}=81, p_{\tau}=15$ and $p_{\zeta}=15$ allowing to synthesize all arguments $i \phi+j \tau+k \zeta$ with $|i| \leq 40,|j| \leq 7$ and $|k| \leq 7$. To save place, the results are given in Fig. 1 to 3 in form of spectra. The short-period terms have been stabilized at the level of five kilometers. At this level, terms up to the argument $21 \phi$ are present (but do not appear in Fig. 1) and each series contains more than 150 terms. In comparison with the first order given in Vienne \& Duriez (1991), we observe that in $\Delta a$, the term in $2 \phi$ is now the major one, like in the results of Taylor et al.(1987).

Concerning the long-period perturbations, Fig. 2 and 3 show that there are only few terms in each solution. All terms larger than $100 \mathrm{~km}$ are already present in the 

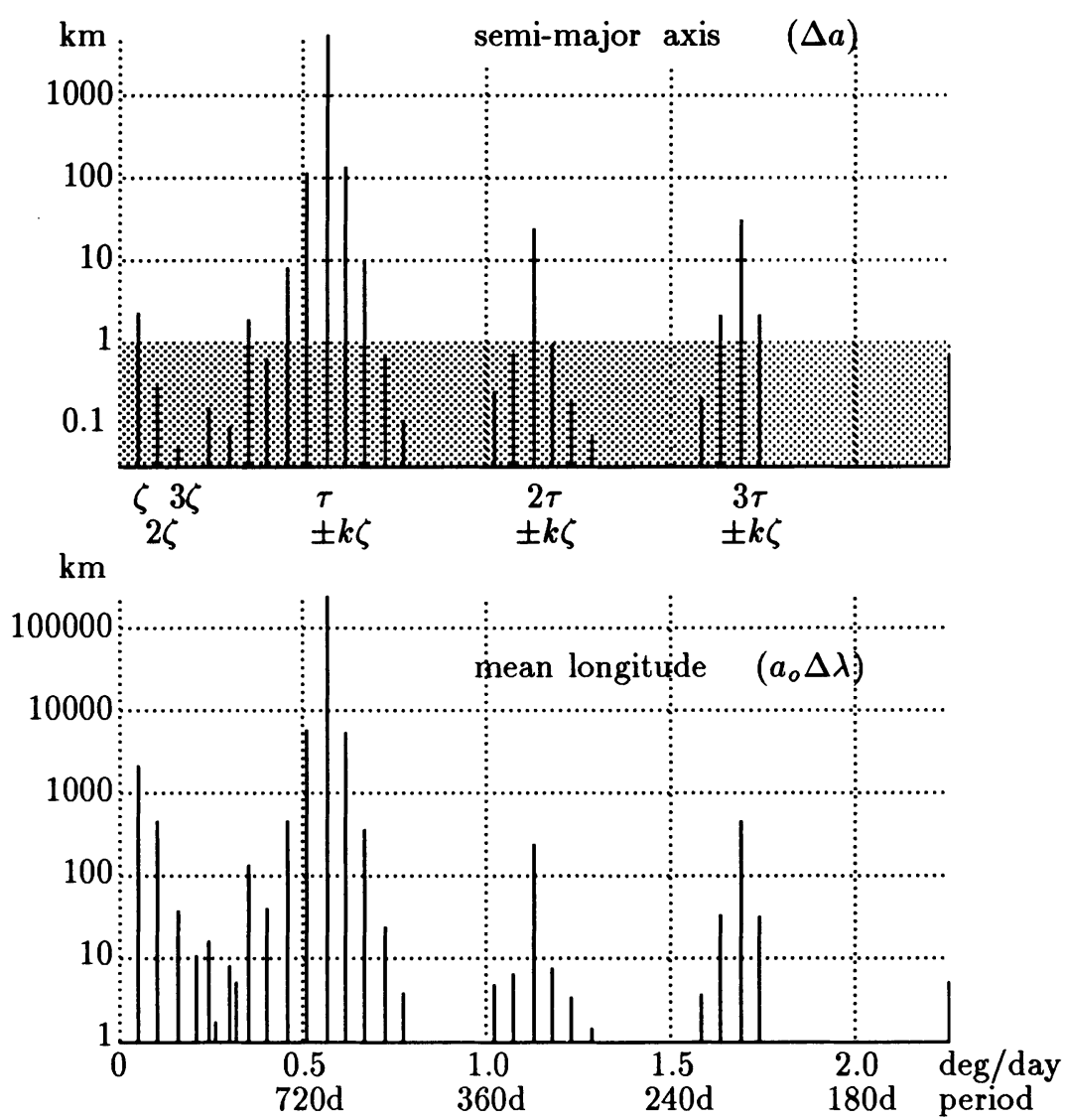

Fig. 2. Long-period perturbations of Hyperion in the semi-major axis and the mean longitude.

current theory of Taylor et al.(1987), except the terms $\tau \pm 2 \zeta$ in $\Delta \lambda$ and $\Delta \varpi$, and $\tau-4 \zeta$ in $\Delta \lambda$. Thus, we see what new terms must be accounted to progress to the kilometer level in precision. We have also to emphasize that the stabilisation of the long-period terms has been reached only at the level of about $50 \mathrm{~km}$ and that requires to compute the semi-major axis with a precision better than $100 \mathrm{~m}$.

At last, we have found the largest perturbations of Titan by Hyperion, which could be detected during the future CASSINI mission if the Hyperion's mass is of the same order than that we have used :

$$
\begin{aligned}
\Delta \lambda^{\prime} & =-0^{\circ} .00127 \sin \tau \quad \Longrightarrow 27 \mathrm{~km} \text { on position } \\
\Delta e^{\prime} & =-0^{\circ} .000012 \cos \zeta \quad \Longrightarrow 15 \mathrm{~km} \\
e^{\prime} \Delta \varpi^{\prime} & =-0^{\circ} .00070 \sin \zeta \quad \Longrightarrow 15 \mathrm{~km}
\end{aligned}
$$



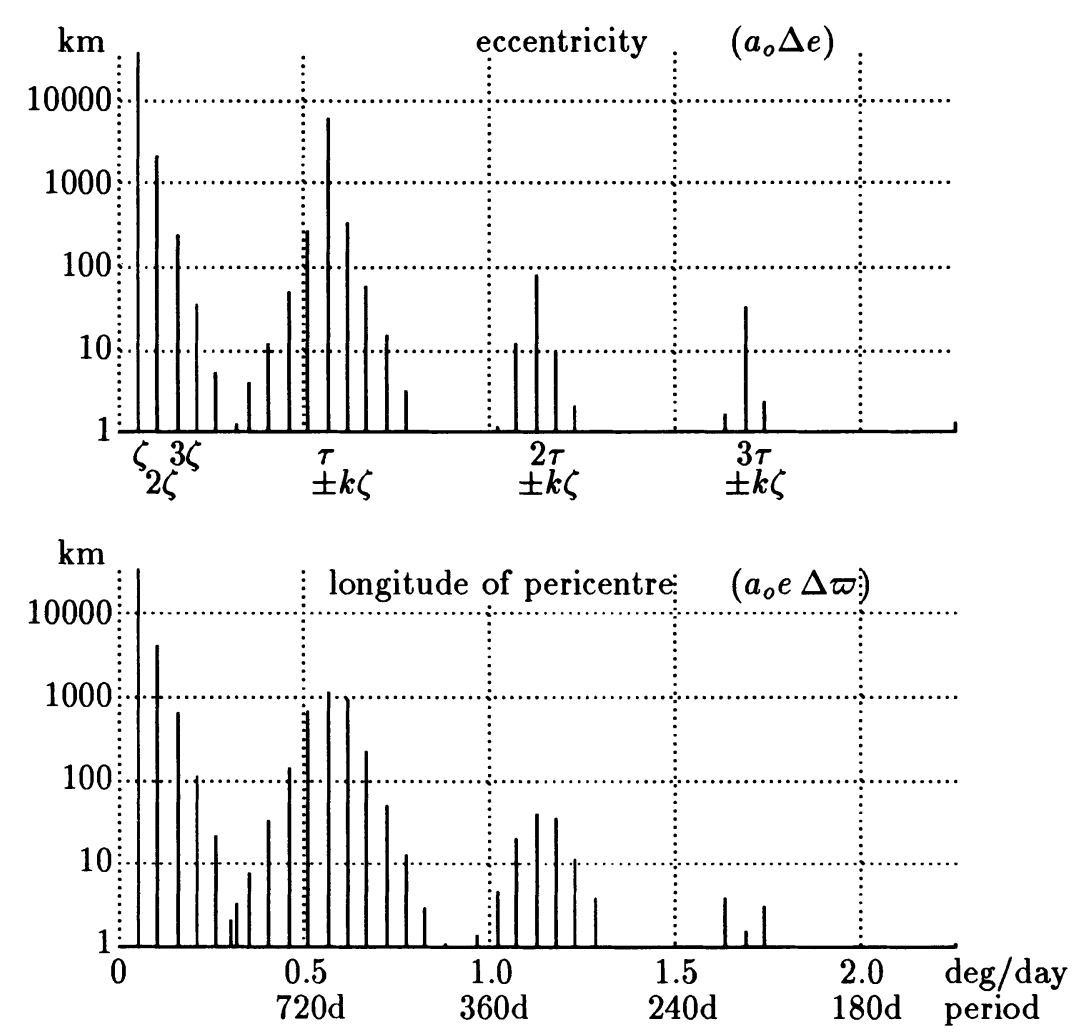

Fig. 3. Long-period perturbations of Hyperion in the eccentricity and the longitude of pericentre

\section{Conclusion}

We have presented a new way to construct by iterations an high order representation for the mutual perturbations of Titan and Hyperion, giving both all short and longperiod terms up to a given precision. However some progress must still be done to reach the kilometer level which will be necessary for the CASSINI mission. We work now in this aim and also to account on the effects of the inclination of the orbits.

\section{References}

Chapront, J., Bretagnon, P., Mehl, M.: 1975, 'Un formulaire pour le calcul des perturbations d'ordre élevé dans les problèmes planétaires', Celes. Mech. 11, 379-399

Message, J.P.: 1989, 'The use of computer algorithms in the construction of a theory of the longperiod perturbations of Saturn's satellite Hyperion', Celes. Mech. 45, 45-53

Taylor, D.B., Sinclair, A.T., Message, P.J.: 1987, 'Corrections to the theory of the orbit of Saturn's satellite Hyperion', A stron. A strophys. 181, 383-390

Vienne, A., Duriez, L.: 1991, 'A general theory of motion for the eight major satellites of Saturn. II: short-period perturbations', Astron. Astrophys. 246, 619-633

Woltjer, J.: 1928, 'The motion of Hyperion', Ann. Sterrewacht Leiden XVI-3, 